\title{
Master Equation Analysis of Thermochemical Nonequilibrium of Nitrogen
}

\author{
Jae Gang Kim* and Iain D. Boyd ${ }^{\dagger}$ \\ University of Michigan, Ann Arbor, MI 48109, USA
}

\begin{abstract}
Using a NASA database of state-to-state transition rates for $\mathrm{N}+\mathrm{N}_{2}$, master equation studies are performed for various nonequilibrium heat bath conditions. In these master equation studies, relaxation of the rotational and vibrational modes, time variation of chemical composition, reaction rate coefficients, and average rotational and vibrational energy losses due to dissociation are each considered in strong and weak nonequilibrium conditions. A system of master equations is coupled with one-dimensional flow equations to analyze the relaxation of $\mathrm{N}_{2}$ in post-normal shock flows. From the results of master equations and the post-normal shock calculations, it is recommended that the rotational nonequilibrium of $\mathrm{N}_{2}$ should be treated as a nonequilibrium mode in hypersonic re-entry calculations.
\end{abstract}

\section{Nomenclature}

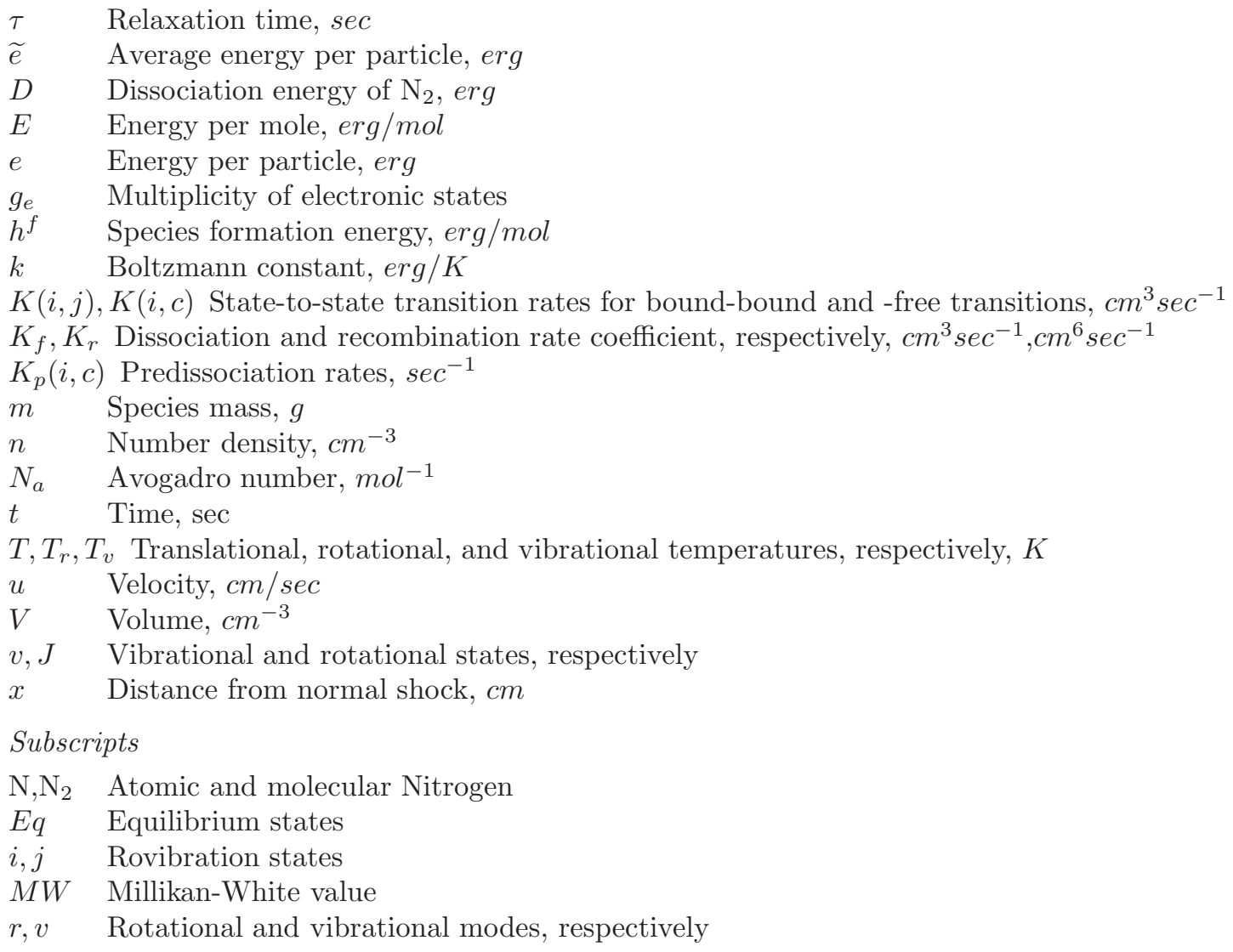

*Post-doctoral Research Fellow, Department of Aerospace Engineering, 1320 Beal Avenue, Member of AIAA.

$\dagger$ Professor, Department of Aerospace Engineering, 1320 Beal Avenue, AIAA Fellow. 


\section{Symbols}

$\epsilon \quad$ Sysmetric factor

$\gamma \quad$ Rovibrational or species concentration, $\mathrm{mol} / \mathrm{g}$

$\hbar \quad$ Planck constant, $\mathrm{erg}-\mathrm{sec}$

$\rho \quad$ Density, $\mathrm{g} / \mathrm{cm}^{3}$

$\xi \quad$ Degree of freedom

\section{Introduction}

$\mathrm{T}^{\mathrm{N}}$ the aerodynamic design of hypersonic vehicles, the prediction of the surface pressure and heat flux on the re-entry module are important. In predicting these surface properties, an accurate thermochemical nonequilibrium model for hypersonic CFD is needed. The widely used model is the two-temperature and multi-temperature models. ${ }^{1}$ In these models, it is assumed that the translational and rotational modes are in equilibrium and these are treated as a trans-rotational temperature, while the other energy modes are treated as nonequilibrium temperatures. However, in recent studies of the high-temperature and strongnonequilibrium gases, uncertainties were observed for these assumptions of the previous thermochemical models.

In $\mathrm{H}_{2}$ mixture gases, the rotational and vibrational relaxations in the electronic ground state of $\mathrm{H}_{2}$ were studied by using the complete sets of state-to-state rates for rotational and vibrational energy transitions by Kim et al. ${ }^{2,3}$ and Kim and Boyd. ${ }^{4}$ In these works, it was observed that the rotational relaxation at low temperature is faster than the relaxation of the vibrational mode. However, at high temperatures above $10,000 \mathrm{~K}$, the rotational and vibrational relaxation times are almost identical. In the analysis of post-normal shock and nozzle expanding flows, the rotational mode of $\mathrm{H}_{2}$ exists in a state of nonequilibrium and the calculated rotational temperatures in these applications are close to the vibration temperatures.

For $\mathrm{N}_{2}+\mathrm{N}_{2}$, the pioneering works by Parker ${ }^{5}$ and Lordi and Mates ${ }^{6}$ presented the effective rotational collision numbers and these were confirmed by the experiments below $1500 \mathrm{~K} .^{7,8}$ However, the limitations on the high-temperature behavior were demonstrated by shock-tube experiments by Sharma and Gillespie ${ }^{9}$ and by Fujita et al. ${ }^{10}$ From the results of Parker and Lordi and Mates, the rotational effective collision number is of the order of 10. This effective collision number of the rotational mode is much smaller than the vibrational mode and it suggests that the rotational mode can be treated as an equilibrium state. However, in the shocktube experiments,, 10 the measured rotational temperature is close to the vibrational temperature. In the work by Park, ${ }^{11}$ the behavior of the rotational mode at high temperatures above $90,000 \mathrm{~K}$ were studied by using existing state-to-state rotational transition rates, which were derived from quantum calculations ${ }^{12}$ and experimental data ${ }^{13,14}$ taken at temperatures equal to or below $1500 \mathrm{~K}$. In this work, it was observed that the calculated rotational relaxation time is larger than the vibrational relaxation time at temperatures above $12,000 \mathrm{~K}$. However, this work is believed to be only incomplete because vibration-rotation coupling is modeled empirically.

For $\mathrm{N}+\mathrm{N}_{2}$, a complete set of state-to-state transition rates was recently calculated by Jaffe et al. ${ }^{15,16}$ at NASA Ames Research Center. The quasi-classical trajectory (QCT) method was adopted to calculate these transition rates based on the new $\mathrm{N}_{3}$ potential energy surfaces. ${ }^{15}$ In the present work, thermochemical nonequilibrium of the rotational and vibrational modes for $\mathrm{N}+\mathrm{N}_{2}$ is studied by master equation calculations with these recent state-to-state transition rates. The master equation studies are performed in various heating and cooling conditions to observe the rotational and vibration behavior in strong-nonequilibrium and high-temperature cases. By coupling the one-dimensional flow equations and a system of master equations, thermochemical nonequilibrium calculations for post-normal shock flows are also performed for various reentry conditions of the Earth.

\section{Master equation study for bound-bound transitions}

For $\mathrm{N}+\mathrm{N}_{2}$, the database of state-to-state transition rates were constructed for 9,390 rovibrational states of the electronic ground-state of $\mathrm{N}_{2}$ by Jaffe et al. ${ }^{15,16}$ In these 9,390 rovibrational states, truly bound and quasi-bound states were included. The QCT method was adopted to evaluate these cross sections and rate coefficients for bound-bound, bound-free, and exchange reactions including the predissociation of quasi-bound states. The database for the $\mathrm{N}+\mathrm{N}_{2}$ system comprises more than 23 million reactions and these 
Table 1. Initial heat bath conditions for bound-bound transitions.

\begin{tabular}{ccc} 
Case & $T(K)$ & $T_{r}=T_{v}(K)$ \\
\hline C1 & 4,000 & 1,000 \\
C2 & 6,000 & 1,000 \\
C3 & 8,000 & 1,000 \\
C4 & 10,000 & 1,000 \\
C5 & 15,000 & 1,000 \\
C6 & 20,000 & 1,000 \\
C7 & 25,000 & 1,000 \\
C8 & 30,000 & 1,000 \\
C9 & 40,000 & 1,000 \\
C10 & 50,000 & 1,000
\end{tabular}

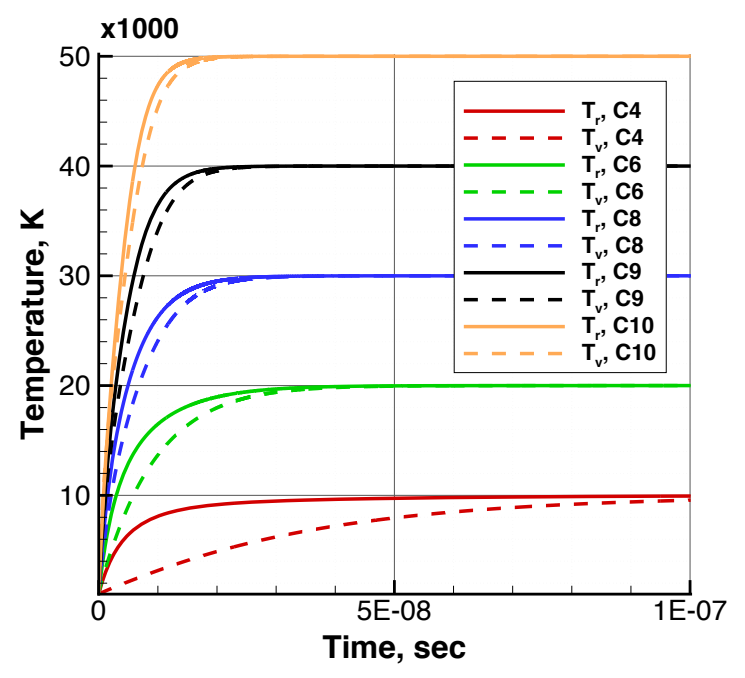

(a) Temperatures.

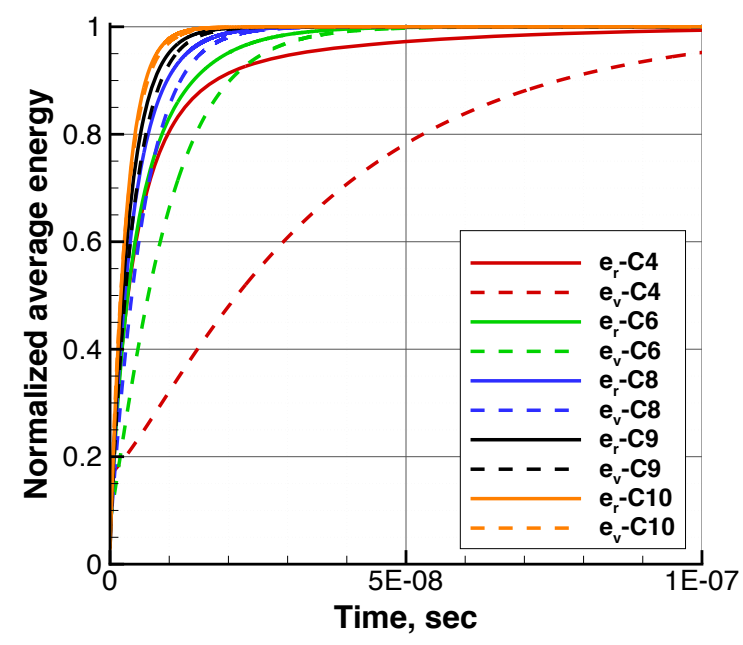

(b) Normalized average energies.

Figure 1. Relaxations of rotational and vibrational temperatures and normalized average energies.

transition rate coefficients were evaluated for translational temperatures of $7,500 K, 10,000 K, 12,500 K$, $15,000 K, 20,000 K$, and $25,000 K$. In the present work, these transition rate coefficients are extended up to a translational temperature of $50,000 \mathrm{~K}$ in order to analyze high-temperature conditions.

By using the database of the transition rates of $\mathrm{N}+\mathrm{N}_{2}$, a system of master equations is constructed to model the relaxations of the rotational and vibrational modes. In this section, the bound-free transition and predissociation are excluded, because the relaxations of the rotational and vibrational modes are mainly produced by the bound-bound transitions. The master equations with this assumption is defined as,

$$
\frac{d n_{i}}{d t}=\sum_{j} K(i, j) n_{x}\left[\frac{Q_{i}}{Q_{j}} n_{j}-n_{i}\right],
$$

where $n_{x}$ is the number density of the colliding species. The rovibrational partition function $Q_{i}$ is defined as

$$
Q_{i}=\epsilon g_{e}(2 J+1) g_{s} \exp \left(-\frac{e_{i}}{k T}\right) .
$$

In $\mathrm{N}_{2}$, the nuclear spin degeneracy $g_{s}$ is 6 when $J$ has an even number and 3 when it is an odd number. The principle of detailed balance between the forward and backward rates is invoked under equilibrium, which 


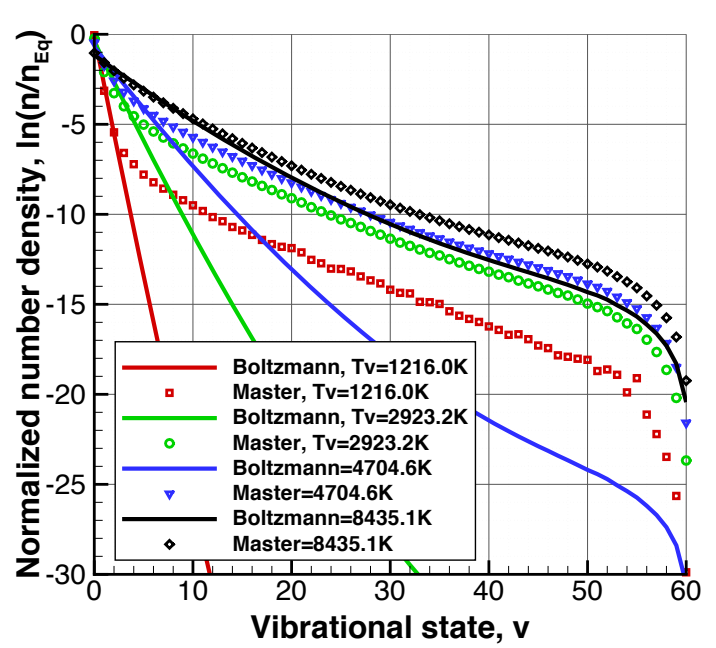

(a) Number density distributions for $\mathrm{N}_{2}(v, 0)$.

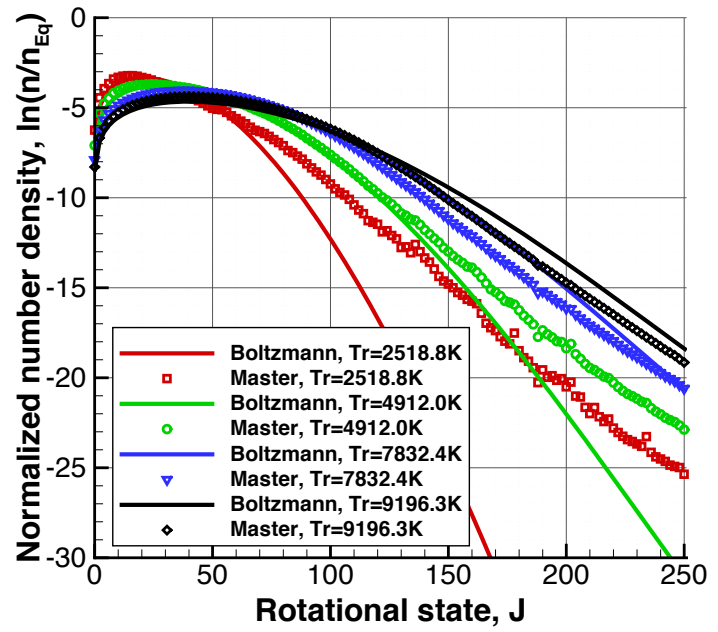

(b) Number density distributions for $\mathrm{N}_{2}(0, J)$.

Figure 2. Normalized number density distributions for C4 case.

leads to

$$
K(i, j) Q_{i}=K(j, i) Q_{j} .
$$

Then, the system of the master equations are constructed by Eq. (1) for all 9,390 rovibrational states of $\mathrm{N}_{2}$.

In integrating the system of master equations, an explicit time integration method is adopted, because the time cost of inverting the matrix of the $\mathrm{N}_{2}$ system is too expensive. In the explicit time integration, for the first five steps, the system of equations is integrated by a 4th order Runge-Kutta-Gill method. ${ }^{17}$ After the 5th step, it is integrated by the Adams-Bashforth-Moulton predictor-corrector method ${ }^{18}$ that increases the accuracy from 6th order to 11th order. After each step of integration, the calculated $n_{i}$ values are modified to conserve mass by distributing the incurred error proportionately to their values. In table 1 , the initial heat bath conditions are tabulated. The temperature range from $4,000 \mathrm{~K}$ to $50,000 \mathrm{~K}$ is studied for weak and strong nonequilibrium conditions. For all cases, the initial number densities of $\mathrm{N}$ and $\mathrm{N}_{2}$ are set to a constant of $5 \times 10^{17} \mathrm{~cm}^{-3}$ and an isothermal condition is applied in these heat bath calculations.

In the present work, energy-equivalent rotational and vibrational temperatures are adopted to characterize the rotational and vibrational modes. These energy-equivalent rotational and vibrational temperatures can be determined by the average rotational and the vibrational energies, respectively. These average energies are defined as

$$
\begin{aligned}
& \widetilde{e}_{r}=\frac{\sum_{i} e_{r}(i) n_{i}}{\sum_{i} n_{i}}, \\
& \widetilde{e}_{v}=\frac{\sum_{i} e_{v}(i) n_{i}}{\sum_{i} n_{i}} .
\end{aligned}
$$

In Fig. 1, the relaxation of the rotational and vibrational temperatures and average energies are shown for various heat bath conditions. In figure (a), it is observed that the relaxation of the rotational mode is discernibly faster than the relaxation of vibrational mode in the $\mathrm{C} 4$ case. However, the rotation relaxation becomes similar to that of vibration when the equilibrium temperature increases. Especially, in cases $\mathrm{C} 8$ to $\mathrm{C} 10$, the rotational and vibrational relaxations are almost identical. In figure (b), the relaxation of average rotational and vibrational energies normalized by equilibrium values are shown. In these average energy relaxations, similar relaxation patterns to those of the temperatures are observed. At high temperatures above $30,000 K$, the energy relaxations of the rotational and vibrational modes are almost identical.

In Fig. 2, rotational and vibrational number density distributions in each relaxation process of the $\mathrm{C} 4$ case are presented. In figure (a), the vibrational number density distributions in $J=0$ states are compared with the Boltzmann distributions according to the nonequilibrium temperatures and in figure (b), the rotation number density distributions in $v=0$ are compared. The nonequilibrium distributions and Boltzmann 


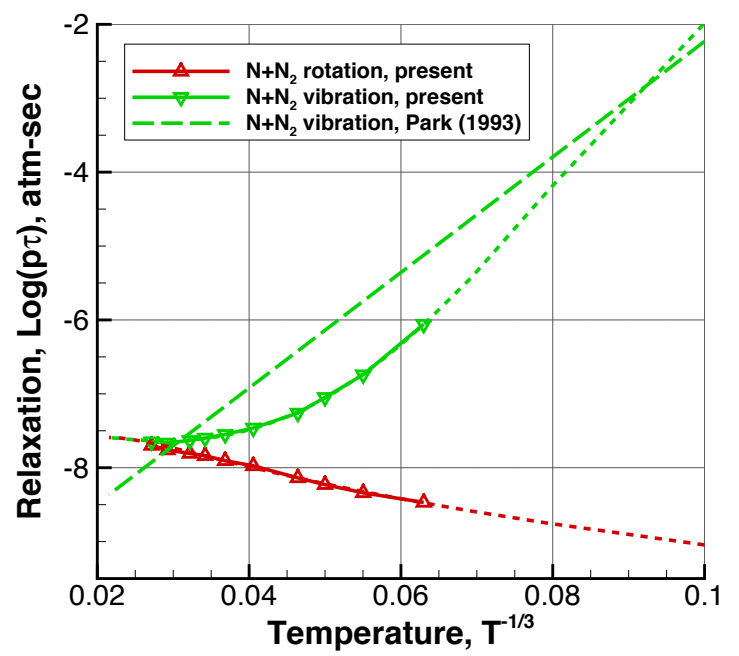

Figure 3. Rotational and vibrational relaxations for $\mathbf{N}+\mathrm{N}_{2}$.

distributions for the rotational and vibrational modes are normalized by their own equilibrium distributions. In figures (a) and (b), for both the rotational and vibrational distributions, the results of the master equation calculations are obviously different from the Boltzmann distributions in strong nonequilibrium conditions. These results show that the energy-equivalent temperature model adopted in the present work has limitations to accurately describe strong nonequilibrium distributions. However, there does not exist an alternative model to accurately describe these nonequilibrium distributions. Also, in the low rotational and vibrational states, where most of the number density exists, the nonequilibrium distributions agree with the Boltzmann distributions.

In Fig. 3, the characteristic relaxation parameters of $p \tau_{r}$ and $p \tau_{v}$ calculated in the present work are compared with Park's model ${ }^{19}$ for $\mathrm{N}+\mathrm{N}_{2}$. In the present work, the relaxation times of the rotational and vibrational modes are defined by using the Landau-Teller form ${ }^{20}$ as

$$
\begin{aligned}
& \frac{\partial \widetilde{e}_{r}}{\partial t}=\frac{\widetilde{e}_{r}(T)-\widetilde{e}_{r}}{\tau_{r}}, \\
& \frac{\partial \widetilde{e}_{v}}{\partial t}=\frac{\widetilde{e}_{v}(T)-\widetilde{e}_{v}}{\tau_{v}},
\end{aligned}
$$

respectively. By equating Eqs. (6) and (7) with the results of the master equation calculations, the relaxation time can be determined with the $e$-folding collision number method. ${ }^{11}$ By multiplying the pressure, one obtains the characteristic rotational and vibrational relaxation parameters $p \tau_{r}$ and $p \tau_{v}$, respectively. The figure shows that the rotational relaxation time is faster than that of vibration initially. However, the differences of relaxation time between the rotational and vibrational modes become small when the temperature increases. At temperatures above $30,000 \mathrm{~K}$, the rotational and vibrational relaxation times are almost identical. In comparison of vibrational relaxation with Park's model, ${ }^{19}$ discernable differences are observed, especially at temperatures above 2,000K. Park's model is based on the empirical equation proposed by Millikan and White, ${ }^{21}$ while the present results are based on the recent $\mathrm{N}_{3}$ potential energy surfaces ${ }^{15}$ calculated by an ab-initio method. 
Table 2. Initial heat bath conditions for bound-free transitions.

\begin{tabular}{cccccc}
\multicolumn{3}{c}{ Heating cases } & \multicolumn{3}{c}{ Cooling cases } \\
\hline Case & $T(K)$ & $T_{r}=T_{v}(K)$ & Case & $T(K)$ & $T_{r}=T_{v}(K)$ \\
\hline H1 & 6,000 & 1,000 & C1 & 6,000 & 30,000 \\
H2 & 8,000 & 1,000 & C2 & 8,000 & 30,000 \\
H3 & 10,000 & 1,000 & C3 & 10,000 & 30,000 \\
H4 & 15,000 & 1,000 & C4 & 15,000 & 30,000 \\
H5 & 20,000 & 1,000 & C5 & 20,000 & 30,000 \\
H6 & 25,000 & 1,000 & & & \\
H7 & 30,000 & 1,000 & & & \\
H8 & 40,000 & 1,000 & & & \\
H9 & 50,000 & 1,000 & & &
\end{tabular}

\section{Master equation study for bound-free transitions}

A system of the master equations with nonequilibrium chemical reactions can be constructed with the state-to-state transition rate coefficients in the following form;

$$
\begin{aligned}
\frac{d n_{i}}{d t}= & \sum_{j} K(i, j) n_{x}\left[\frac{Q_{i}}{Q_{j}} n_{j}-n_{i}\right] \\
& +K(i, c) n_{x}\left[\frac{Q_{i} Q_{t_{N_{2}}}}{Q_{N}^{2} Q_{t_{N}}^{2}} \exp \left(\frac{D_{i}}{k T}\right) n_{N}^{2}-n_{i}\right] \\
& +K_{p}(i, c)\left[\frac{Q_{i} Q_{t_{N_{2}}}}{Q_{N}^{2} Q_{t_{N}}^{2}} \exp \left(\frac{D_{i}}{k T}\right) n_{N}^{2}-n_{i}\right] \\
\frac{d n_{N}}{d t}= & 2 \sum_{i} K(i, c) n_{x}\left[n_{i}-\frac{Q_{i} Q_{t_{N_{2}}}}{Q_{N}^{2} Q_{t_{N}}^{2}} \exp \left(\frac{D_{i}}{k T}\right) n_{N}^{2}\right] \\
& +2 \sum_{i} K_{p}(i, c)\left[n_{i}-\frac{Q_{i} Q_{t_{N_{2}}}}{Q_{N}^{2} Q_{t_{N}}^{2}} \exp \left(\frac{D_{i}}{k T}\right) n_{N}^{2}\right],
\end{aligned}
$$

where the translational partition function $Q_{t}$ is defined as

$$
Q_{t}=V\left(\frac{m k T}{\hbar}\right)^{3 / 2} \text {. }
$$

The principle of detailed balancing relations for bound-free transitions are

$$
K(i, c) \frac{Q_{i} Q_{t_{N_{2}}}}{Q_{N}^{2} Q_{t_{N}}^{2}} \exp \left(\frac{D_{i}}{k T}\right)=K(c, i) .
$$

By using Eqs. (8) and (11), a system of 9,391 master equations are constructed for 9,390 rovibrational states of $\mathrm{N}_{2}$ and atomic species $\mathrm{N}$.

In the computational method, an implicit time integration method accurate to third order along the diagonal and second order off-diagonal elements is adopted in integrating the 9,391 ordinary differential equations of Eqs. (8) and (9). In integrating these master equations, the implicit method is more time consuming than the explicit method. However, in chemical reacting calculations, the implicit technqieu is required due to the matrix stiffness.

The variation in number density of $n_{N_{2}}$ and $n_{N}$ are calculated for heating and cooling environments in the isothermal heat bath conditions. In table 2, these initial heat bath conditions are tabulated. The temperatures range from $6,000 \mathrm{~K}$ to $50,000 \mathrm{~K}$ in heating cases and from $6,000 \mathrm{~K}$ to $20,000 \mathrm{~K}$ in cooling cases, and are studied for weak and strong nonequilibrium conditions. For all cases, initial number densities of $\mathrm{N}$ and $\mathrm{N}_{2}$ are set to a constant of $1 \times 10^{18} \mathrm{~cm}^{-3}$. 


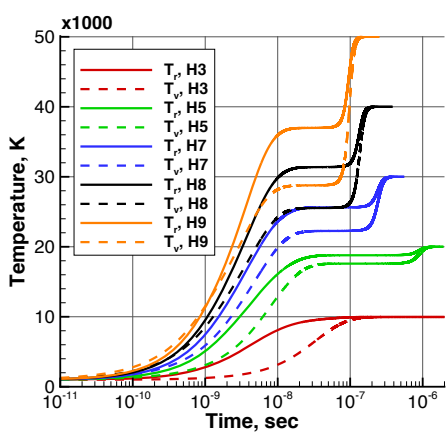

(a) Temperatures.

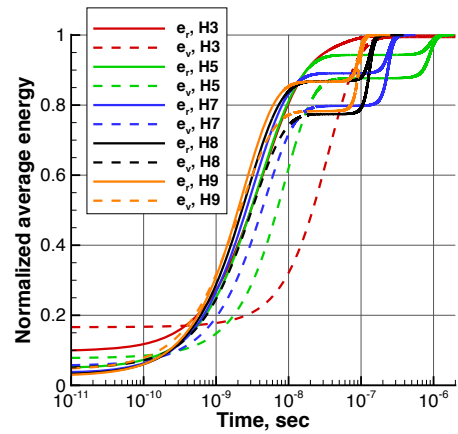

(b) Normalized average energies.

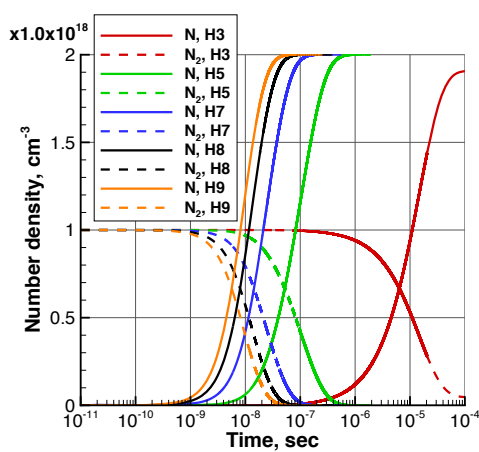

(c) Species number densities.

Figure 4. Relaxations of rotational and vibrational temperatures, normalized average energies, and species number densities in heating conditions.

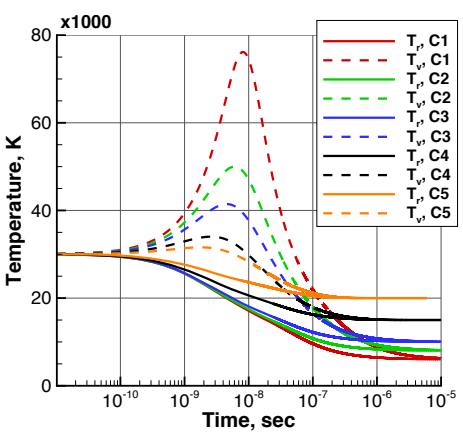

(a) Temperatures.

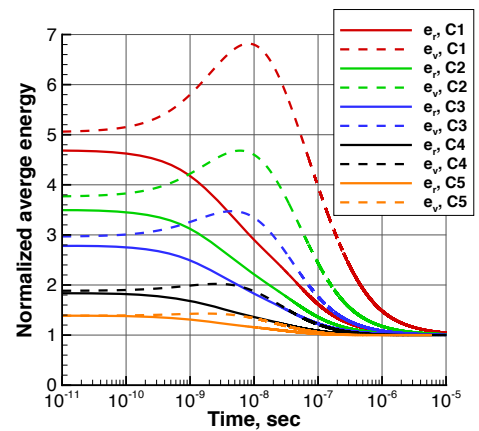

(b) Normalized average energies.

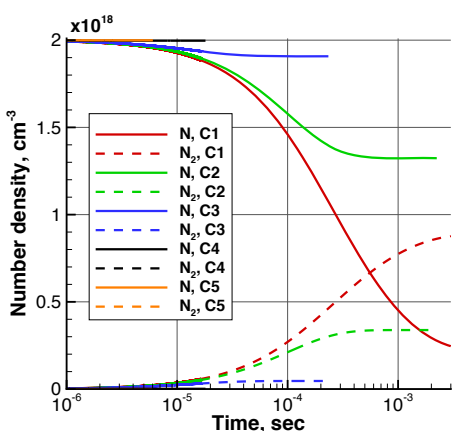

(c) Species number densities.

Figure 5. Relaxations of rotational and vibrational temperatures, normalized average energies, and species number densities in cooling conditions.

In Fig. 4, the relaxations of the rotational and vibrational temperatures, normalized average energies, and species number densities are presented for heating cases. Except for the H4 case, most of the chemical reactionsoccur in the rotational and vibrational nonequilibrium processes and these nonequilibrium processes are in a quasi-steady state (QSS) of rotational and vibrational energies. During this QSS period, the rotational and vibrational temperatures and energies are at almost constant values, and the chemical reactions occur rapidly. In the QSS period, the number density rate of change on the left-hand side in Eq. (8) is much smaller than both the sum of all incoming rates and sum of all outgoing rates on the right-hand side. This phenomenon results in the rotational and vibrational energies maintaining near-constant values.

In Fig. 5, the relaxations of the rotational and vibrational temperatures, normalized average energies, and species number densities by master equation calculations are shown for the cooling cases. In these figures, it is observed that the vibrational temperature and average energy are locally increased. This is because, in this local period, the number density of high-vibrational energy level molecules is increased due to recombination. This local increasing phenomenon was observed in the previous thermochemical nonequilibrium studies of $\mathrm{H}_{2}{ }^{2-}$. ${ }^{4}$ From the figures (a) to (c), it is also observed that the recombination of $\mathrm{N}_{2}$ by three-body collisions of $\mathrm{N}+\mathrm{N}+\mathrm{N}$ mostly occur when there is equilibrium of the rotational and vibrational modes.

In the results of the master equation calculations for heating and cooling conditions, the reaction rate coefficients of dissociation and recombination are derived as follows;

$$
\begin{gathered}
-\frac{d\left[N_{2}\right]}{d t}=K_{f}\left[N_{2}\right] n_{x}, \\
-\frac{d[N]}{d t}=2 K_{r}[N]^{2} n_{x} .
\end{gathered}
$$




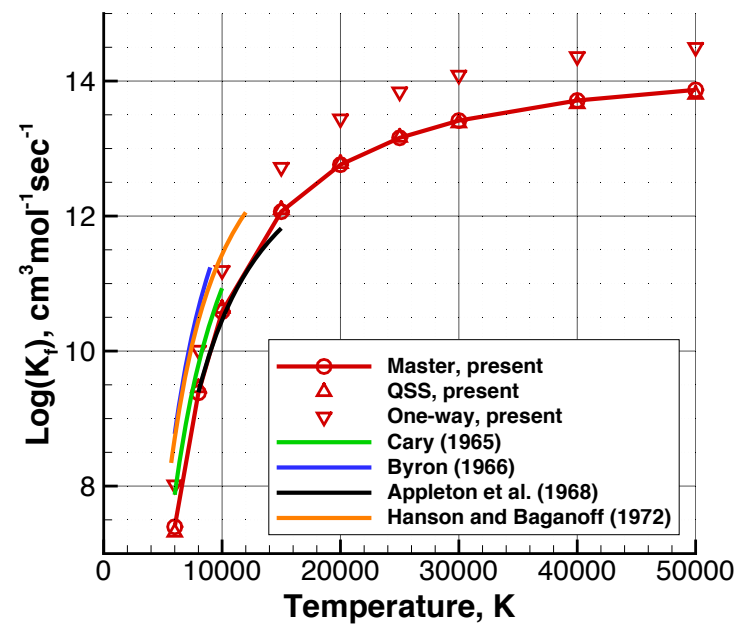

(a) Dissociation reaction rate coefficients.

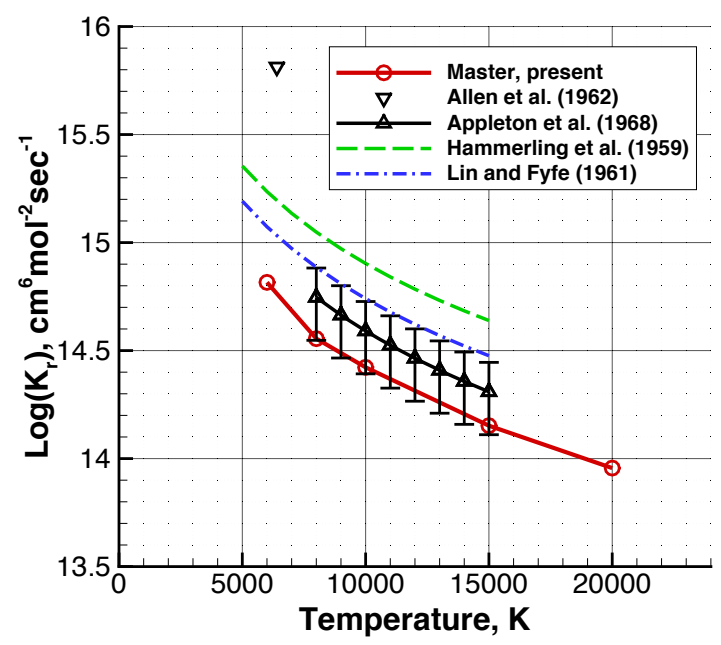

(b) Recombination reaction rate coefficients.

Figure 6. Comparisons of the calculated reaction rate coefficients with the shock-tube experiments ${ }^{22-26}$ and $^{2}$ estimated data. ${ }^{27,28}$

In the dissociation process for heating conditions, the reaction occurs in QSS of the rotational and vibrational modes. The dissociation reaction rate coefficients in this QSS period can be derived by solving the set of algebraic equations by using the QSS assumption. The rate of change of $n_{i}$ is defined as

$$
\frac{d n_{i}}{d t}=\sum_{j} K(j, i) n_{j} n_{x}-\sum_{j} K(i, j) n_{i} n_{x}-K(i, c) n_{i} n_{x}+K(c, i) n_{N}^{2} n_{x}-K_{p}(i, c) n_{i}+K_{p}(c, i) n_{N}^{2} .
$$

From the assumption of QSS of rotational and vibrational energies, the left-hand side of Eq. (14) can be set to be zero. By using normalized populations, $\phi_{i}=n_{i} / n_{i_{E} q}, \phi_{j}=n_{j} / n_{j_{E} q}$, and $\phi_{N}=n_{N} / n_{N_{E} q}$, Eq. (14) can be rewritten as

$$
\left[\sum_{j} K(i, j)+K(i, c)+\frac{K_{p}(i, c)}{n_{x}}\right] \phi_{i}-\sum_{j} K(i, j) \phi_{j}=\left[K(i, c)+\frac{K_{p}(i, c)}{n_{x}}\right] \phi_{N}^{2} .
$$

On the other hand, the sum of all $n_{i}$ must equal the given number density of $\mathrm{N}_{2}$; that is,

$$
\sum_{i} n_{i_{E q}} \phi_{i}=n_{N_{2_{E q}}} \chi
$$

In Eq. (15), we drop the first equation and substitute using Eq. (16). This approach is based on the intuition that the QSS relation is least likely to be satisfied by the rovibrational ground state of $\mathrm{N}_{2}$. Then Eq. (15) can be rewritten as a $9,390 \times 9,390$ matrix;

$$
\mathbf{M} \vec{\phi}=\vec{C} \phi_{N}^{2}+\vec{D} \chi
$$

The normalized population vector $\vec{\phi}$ can be defined by using homogeneous $\vec{\phi}_{h}$ and particular solutions $\vec{\phi}_{p}$ as

$$
\vec{\phi}=\vec{\phi}_{h}+\vec{\phi}_{p} \phi_{N}^{2}
$$

Then, the homogeneous and particular solutions are

$$
\begin{aligned}
\vec{\phi}_{h} & =\mathbf{M}^{-1} \vec{D} \chi, \\
\vec{\phi}_{p} & =\mathbf{M}^{-1} \vec{C},
\end{aligned}
$$




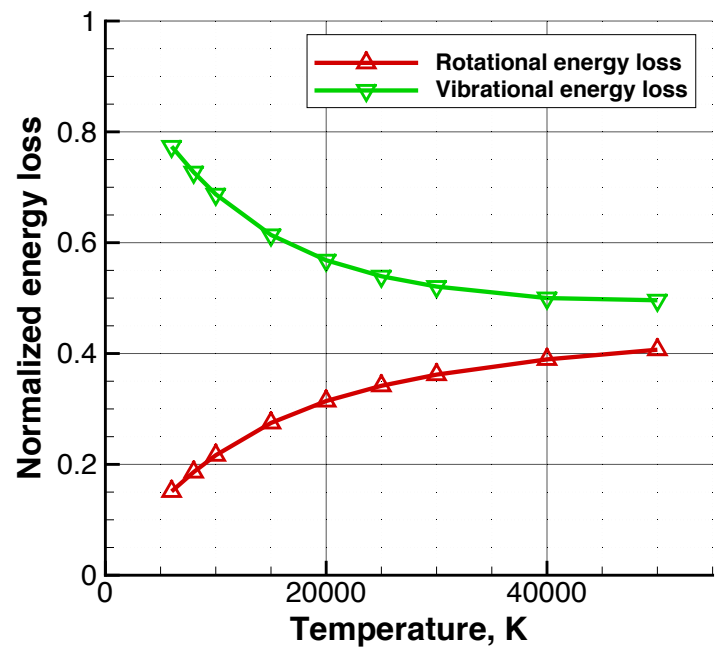

Figure 7. Rotational and vibrational energy losses due to dissociation.

respectively. By using the normalized populations, the rate of change of $n_{N}$ can be rewritten from Eq. (9) as

$$
\frac{d n_{N}}{d t}=2 n_{N_{2}} n_{x} \sum_{i} K(i, c) \frac{Q_{i}}{Q_{m}}\left[\phi_{i}+\phi_{N}^{2}\right]+2 n_{N_{2}} \sum_{i} K_{p}(i, c) \frac{Q_{i}}{Q_{m}}\left[\phi_{i}+\phi_{N}^{2}\right],
$$

where $Q_{m}$ is the molecular partition function of $\mathrm{N}_{2}$. By using the homogeneous and particular solutions of Eq. (18), this equation can be derived as follows;

$$
\begin{aligned}
\frac{d n_{N}}{d t}= & n_{N_{2}} n_{x} \sum_{i} 2\left[K(i, c)+\frac{K_{p}(i, c)}{n_{x}}\right] \frac{Q_{i}}{Q_{m}} \phi_{i, h} \\
& -n_{N}^{2} n_{x} \sum_{i} 2\left[K(i, c)+\frac{K_{p}(i, c)}{n_{x}}\right] \frac{Q_{i}}{Q_{m}} \frac{n_{N_{2_{E q}}}}{n_{N_{E q}}^{2}}\left[1-\phi_{i, p}\right] .
\end{aligned}
$$

Then, we can define the dissociation and recombination reaction rate coefficients in the QSS as

$$
\begin{aligned}
K_{f_{Q S S}} & =2 \sum_{i}\left[K(i, c)+\frac{K_{p}(i, c)}{n_{x}}\right] \frac{Q_{i}}{Q_{m}} \phi_{i, h}, \\
K_{r_{Q S S}} & =2 \sum_{i}\left[K(i, c)+\frac{K_{p}(i, c)}{n_{x}}\right] \frac{Q_{i}}{Q_{m}} \frac{n_{N_{2_{E q}}}}{n_{N_{E q}}^{2}}\left[1-\phi_{i, p}\right] .
\end{aligned}
$$

In the master equation calculations for the cooling cases, most recombined molecules are generated in the equilibrium states. This means that the QSS assumption for the recombination process of $\mathrm{N}+\mathrm{N}+\mathrm{N}$ is not applicable. In the present work, the dissociation reaction rate of QSS is calculated by using Eq. (23).

In Fig. 6, the calculated reaction rate coefficients for dissociation and recombination are compared with shock-tube ${ }^{22-26}$ and theoretically estimated data. ${ }^{27,28}$ In the comparisons of the dissociation reaction rates of figure (a), the present reaction rates derived from the results of the master equations by using Eq. (12) have similar values with the reaction rates calculated by QSS assumptions. However, these reaction rates have discernable differences with the one-way rates. ${ }^{1}$ The one-way rate in the present work is defined as

$$
K_{f_{\text {one-way }}}=\frac{\sum_{i} K(i, c) Q_{i}}{\sum_{i} Q_{i}}
$$

In the one-way rates, the nonequilibrium effects of bound-bound transitions of rotational and vibrational modes cannot be presented. This limitation makes the discernable differences between the one-way reaction 
rates and reaction rates by QSS assumption and results of master equation calculations. In the comparisons with the shock-tube data, the present reaction rates by QSS assumption and results of master equation calculations have similar values with the experiments by Appleton et al. ${ }^{24}$ In figure (b), the comparison of the recombination reaction rates is presented. This figure shows that the present reaction rates of recombination process fall within the error bar of the shock-tube experiments by Appleton et al. ${ }^{24}$

In Fig. 7, the rotational and vibrational average energy losses due to dissociation are presented. The average energy loss in the present work is defined as

$$
\widetilde{e}_{x_{\text {loss }}}=\frac{\sum_{i}\left[K(i, c) n_{x}+K_{p}(i, c)\right] e_{x}(i) n_{i_{E q}}\left[\phi_{N}^{2}-\phi_{i}\right]}{\sum_{i}\left[K(i, c) n_{x}+K_{p}(i, c)\right] n_{i_{E q}}\left[\phi_{N}^{2}-\phi_{i}\right]},
$$

where $x$ represents rotational or vibrational modes. This average energy loss is normalized by the dissociation energy of $\mathrm{N}_{2}$. In the figure, it is observed that the normalized vibrational energy loss is about 0.8 at $10,000 \mathrm{~K}$ and this value is approaches 0.5 when the temperature increases up to $50,000 \mathrm{~K}$. In the normalized rotational energy loss, the value is about 0.25 at $10,000 K$ and approaches 0.4 when the temperature increases. This is because, at low temperatures, most of the dissociation occurs at high-vibrational and low-rotational energy levels. When the temperature increases, the average dissociated energy levels move to lower vibrational and higher rotational energy levels.

\section{1-D post-normal shock flow calculations}

The system of master equations are coupled with one-dimensional flow equations to analyze the thermochemical nonequilibrium of $\mathrm{N}_{2}$ in 1-D post-normal shock flows. In the present work, electron collision, radiative heating, and electronic excitation and ionization of heavy particle are ignored to observe the rotational and vibrational relaxations and chemical reactions by heavy particle collisions. Pure neutral $\mathrm{N}$ and $\mathrm{N}_{2}$ in the electronic ground state is considered in the flow calculations. The mass, momentum, and energy conservation equations in the steady-state are derived as

$$
\begin{aligned}
\frac{1}{\rho} \frac{d \rho}{d x} & =-\frac{1}{u^{2}} \frac{d}{d x}\left(\frac{u^{2}}{2}\right), \\
\frac{d}{d x}\left(\frac{u^{2}}{2}\right) & =-\frac{1}{\rho} \frac{d p}{d x}, \\
u \frac{d h}{d x} & =0,
\end{aligned}
$$

where, the pressure $p\left(\right.$ dyne $\left./ \mathrm{cm}^{2}\right)$ and enthalpy $h(\mathrm{erg} / \mathrm{g})$ are defined as

$$
\begin{gathered}
p=\sum_{s}^{N, N_{2}} \rho N_{a} \gamma_{s} k T, \\
h=\sum_{s}^{N, N_{2}} h_{s}^{f} \gamma_{s}+\frac{5}{2} N_{a} k T \sum_{s}^{N, N_{2}} \gamma_{s}+\left(E_{r}+E_{v}\right) \gamma_{H_{2}}+\frac{u^{2}}{2},
\end{gathered}
$$

respectively. The species conservation equations of $\mathrm{N}_{2}+\mathrm{N}_{2}$ and $\mathrm{N}+\mathrm{N}_{2}$ can be written as follows;

$$
\begin{aligned}
u \frac{d \gamma_{N}}{d x}= & -2\left(\frac{d \gamma_{N_{2}}}{d t}\right) \\
u \frac{d \gamma_{N_{2}}}{d x}= & -K_{f_{N_{2}+N_{2}}} \rho N_{a} \gamma_{N_{2}}^{2}+K_{r_{N_{2}+N_{2}}} \rho^{2} N_{a}^{2} \gamma_{N}^{2} \gamma_{N_{2}} \\
& +\sum_{i} K(i, c)\left[\frac{Q_{i} Q_{t_{N_{2}}}}{Q_{N}^{2} Q_{t_{N}}^{2}} \exp \left(\frac{D_{i}}{k T}\right) \rho N_{a} \gamma_{N}^{2}-\gamma_{i}\right] \rho N_{a} \gamma_{N} \\
& +\sum_{i} K_{p}(i, c)\left[\frac{Q_{i} Q_{t_{N_{2}}}}{Q_{N}^{2} Q_{t_{N}}^{2}} \exp \left(\frac{D_{i}}{k T}\right) \rho N_{a} \gamma_{N}^{2}-\gamma_{i}\right]
\end{aligned}
$$

In the present work, the two-temperature model of the dissociation reaction $K_{f}$ is adopted for $\mathrm{N}_{2}+\mathrm{N}_{2}$. This is because there does not exist a model to describe the rotational nonequilibrium effect in dissociation processes 


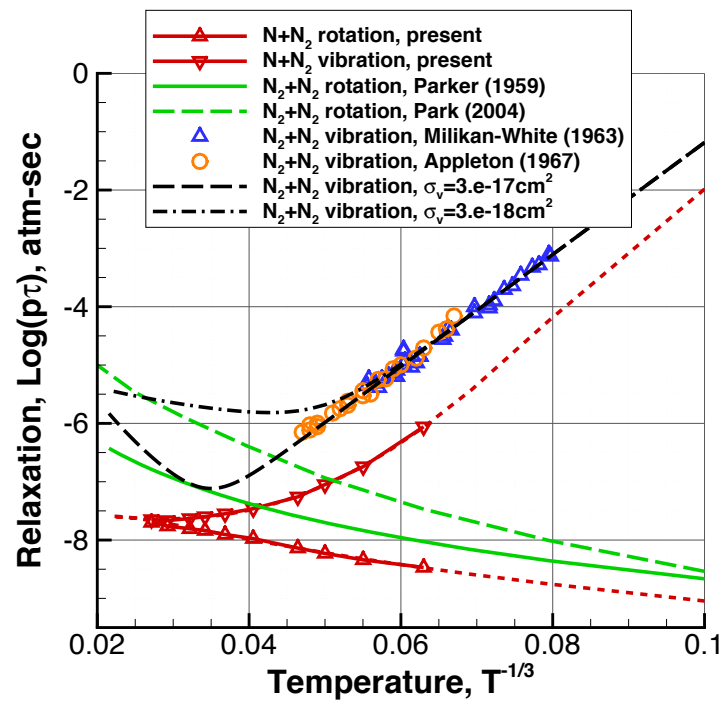

Figure 8. Comparisions of rotational and vibrational relaxations between $\mathbf{N}+\mathbf{N}_{2}$ and $\mathbf{N}_{2}+\mathbf{N}_{2}$.

for $\mathrm{N}_{2}+\mathrm{N}_{2}$. However, in the other reaction process for $\mathrm{N}+\mathrm{N}_{2}$, the rotational and vibrational nonequilibrium effects are fully considered by coupling the system of master equations. In the present work, the $K_{f}$ proposed by Baulch et al. ${ }^{29}$ is adopted and this reaction rate is $K_{f_{N_{2}+N_{2}}}=6.1 \times 10^{-3} T_{a}^{-1.6} \exp \left(-113200 / T_{a}\right)$. The value for $K_{r}$ is determined from the relation with the equilibrium constant as $K e=K_{f} / K_{r}$. In describing the rotation-to-vibration energy transfer by $\mathrm{N}_{2}+\mathrm{N}_{2}$, Park's model ${ }^{11}$ is adopted. The rotation-to-vibration energy transfer by $\mathrm{N}+\mathrm{N}_{2}$ is described by coupling the system of the master equations. Then, the rotational and vibrational energy relaxations are evaluated by using the following equations;

$$
\begin{aligned}
& u \frac{d E_{r}}{d x}=\frac{\widetilde{e}_{r}(T) N_{a} \gamma_{N_{2}}-E_{r}}{\tau_{r}}+f_{R V} \frac{E_{v}-\widetilde{e}_{v}\left(T_{r}\right) N_{a} \gamma_{N_{2}}}{\tau_{M W}}+\Psi_{r} D_{N_{2}} N_{a}\left(\frac{d \gamma_{N_{2}}}{d t}\right)_{N_{2}+N_{2}} \\
& +\sum_{i} e_{r_{i}}\left[\begin{array}{c}
\sum_{j} K(i, j)\left[\frac{Q_{i}}{Q_{j}} \gamma_{j}-\gamma_{i}\right] \rho N_{a}^{2} \gamma_{N} \\
+K(i, c)\left[\frac{Q_{i} Q_{t_{N_{2}}}}{Q_{N}^{2} Q_{t_{N}}^{2}} \exp \left(\frac{D_{i}}{k T}\right) \rho N_{a} \gamma_{N}^{2}-\gamma_{i}\right] \rho N_{a}{ }^{2} \gamma_{N} \\
+K_{p}(i, c)\left[\frac{Q_{i} Q_{t_{N}}}{Q_{N}^{2} Q_{t_{N}}^{2}} \exp \left(\frac{D_{i}}{k T}\right) \rho N_{a} \gamma_{N}^{2}-\gamma_{i}\right] N_{a}
\end{array}\right], \\
& u \frac{d E_{v}}{d x}=\left(1-f_{R V}\right) f_{D} \frac{\widetilde{e}_{v}(T) N_{a} \gamma_{N_{2}}-E_{v}}{\tau_{M W}+\tau_{c}}+f_{R V} \frac{\widetilde{e}_{v}\left(T_{r}\right) N_{a} \gamma_{N_{2}}-E_{v}}{\tau_{M W}}+\Psi_{v} D_{N_{2}} N_{a}\left(\frac{d \gamma_{N_{2}}}{d t}\right)_{N_{2}+N_{2}} \\
& +\sum_{i} e_{v_{i}}\left[\begin{array}{c}
\sum_{j} K(i, j)\left[\frac{Q_{i}}{Q_{j}} \gamma_{j}-\gamma_{i}\right] \rho N_{a}^{2} \gamma_{N} \\
+K(i, c)\left[\frac{Q_{i} Q_{t_{N}}}{Q_{N}^{2} Q_{t_{N}}^{2}} \exp \left(\frac{D_{i}}{k T}\right) \rho N_{a} \gamma_{N}^{2}-\gamma_{i}\right] \rho N_{a}^{2} \gamma_{N} \\
+K_{p}(i, c)\left[\frac{Q_{i} Q_{t_{N}}}{Q_{N}^{2} Q_{t_{N}}^{2}} \exp \left(\frac{D_{i}}{k T}\right) \rho N_{a} \gamma_{N}^{2}-\gamma_{i}\right] N_{a}
\end{array}\right] .
\end{aligned}
$$

In Park's model, ${ }^{11}$ the fractional contribution of the rotation-to-vibration energy transfer to the total energy transfer for $\mathrm{N}_{2}+\mathrm{N}_{2}$ is set to a constant of 0.4 and this value is derived from $f_{R V}=k T /(k T+1.5 k T)$. In the present work, unlike Park's model, the fractional contribution $f_{R V}$ is determined by

$$
f_{R V}=\frac{\left(\xi_{v} / 2\right) k T}{\left(\xi_{r} / 2\right) k T+1.5 k T} .
$$

The normalized rotational and vibrational energy losses, $\Psi_{r}$ and $\Psi_{v}$ are set to constants of 0.4 and 0.5 , respectively. From the results of master equation calculations in Section III, we observe that the rotational 


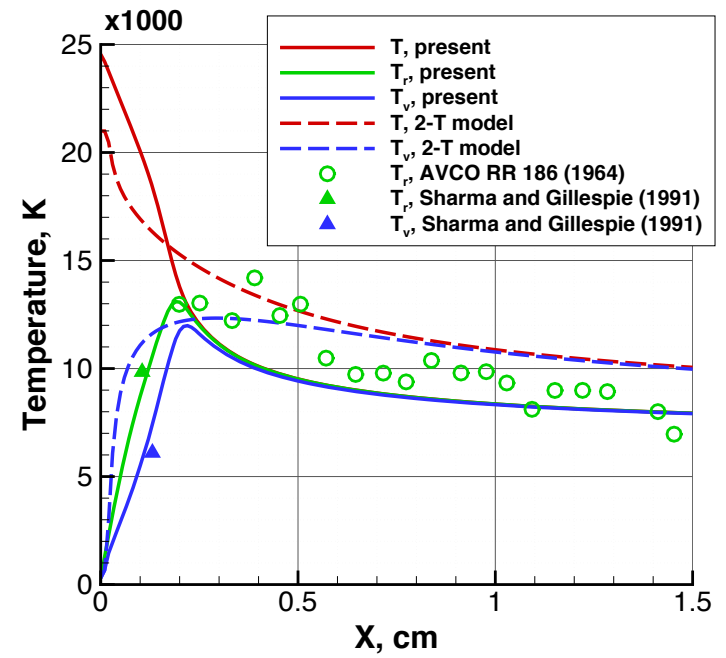

(a) Rotational and vibrational temperatures.

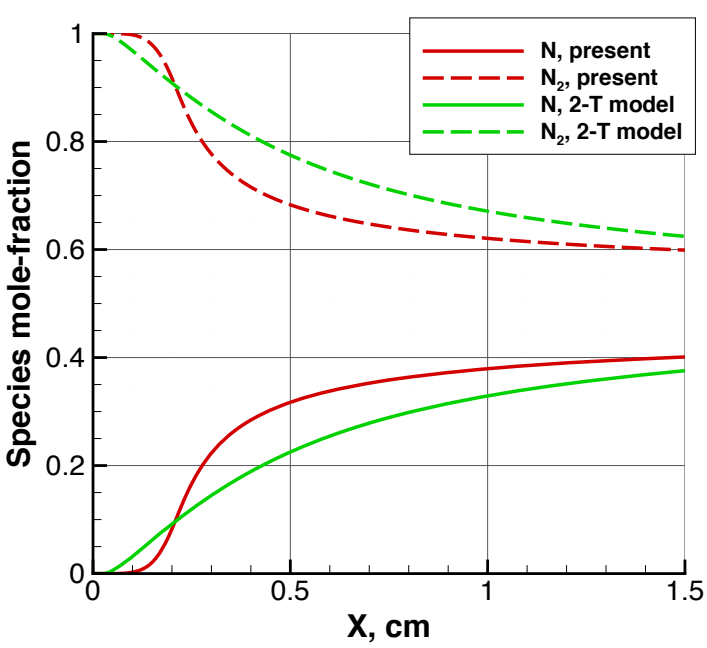

(b) Species mole-fractions.

Figure 9. Comparisons of the present rotational and vibrational temperatures and species mole-fractions with the shock-tube data by Sharma and Gillespie ${ }^{9}$ and $\mathrm{AVCO}^{32}$ and with the calculated results of 2-T model.

and vibrational losses approach these values. From the recent master equation studies for $\mathrm{H}_{2}{ }^{2-},{ }^{4}$ the energy losses approach similar values as 0.35 and 0.5 for the rotational and vibrational modes, respectively. The diffusion correction factor ${ }^{1} f_{D}$ in vibration-to-translation transfer is defined as

$$
f_{D}=\left|\frac{T_{s}-T_{v}}{T_{s}-T_{v s}}\right|^{s-1}
$$

where $s$ is an arbitrary parameter given as $3.5 \times \exp \left(-5,000 / T_{s}\right)$ for $\mathrm{N}_{2} . T_{s}$ and $T_{v s}$ are the translational and vibrational temperatures immediately behind the shock wave. In Eqs. (34) and (35), the unknown variable is the rovibrational concentration $\gamma_{i}$. Unfortunately, we cannot directly calculate $\gamma_{i}$ from the state-to-state kinetics, because of the lack of knowledge about the state-to-state transition rates for $\mathrm{N}_{2}+\mathrm{N}_{2}$. In the present work, this rovibrational concentration $\gamma_{i}$ is determined from the Boltzmann distributions according to the nonequilibrium temperatures as follows;

$$
\begin{aligned}
\gamma_{i} & =\frac{\eta\left(i, T_{r}, T_{v}\right)}{\sum_{i} \eta\left(i, T_{r}, T_{v}\right)} \gamma_{N_{2}}, \\
\eta\left(i, T_{r}, T_{v}\right) & =\epsilon g_{e} g_{s} J(J+1) \exp \left[-\frac{e_{r}(i)}{k T_{r}}-\frac{e_{v}(i)}{k T_{v}}\right] .
\end{aligned}
$$

In Fig. 8, the rotational and vibrational relaxation parameters for $\mathrm{N}+\mathrm{N}_{2}$ and $\mathrm{N}_{2}+\mathrm{N}_{2}$ are compared. In rotational relaxations for $\mathrm{N}_{2}+\mathrm{N}_{2}$, the rotational relaxation by Parker ${ }^{5}$ is evaluated theoretically for the rigid-rotor model and is much faster than the rotational relaxation of Park. ${ }^{11}$ The rotational relaxation by Park is obtained from the existing state-to-state rotational transition rate coefficients with the expression proposed by Rahn and Palmer. ${ }^{30}$ In the present work, rotational relaxation proposed by Park is adopted to calculate the post-normal shock flows. In the vibrational relaxations for $\mathrm{N}_{2}+\mathrm{N}_{2}$, the collision limited relaxation time ${ }^{1} \tau_{c}$ is added to the Millikan-White relaxation time ${ }^{21} \tau_{M W}$ to account for the fact that the vibrational excitation rates can not exceed the elastic collision rates. This collision limited relaxation time is defined as

$$
\tau_{c}=\sigma_{v}\left(\frac{50,000}{T}\right)^{2}
$$

where $\sigma_{v}$ varies from $\sim 10^{16}$ to $\sim 10^{18} \mathrm{~cm}^{2}$. The widely used value is $3.0 \times 10^{17} \mathrm{~cm}^{2}$. However, the vibrational relaxation time with the collision limiting cross section of $3.0 \times 10^{17} \mathrm{~cm}^{2}$ is so much faster than that of Park's 
Table 3. Freestream conditions for 1-D post-normal shock calculations.

\begin{tabular}{ccccccc} 
Case & $p_{\infty}($ Torr $)$ & $T_{\infty}(\mathrm{K})$ & $\rho_{\infty}\left(\mathrm{g} / \mathrm{cm}^{3}\right)$ & $h_{\infty}(\mathrm{MJ} / \mathrm{kg})$ & $u_{\infty}(\mathrm{km} / \mathrm{sec})$ & $M_{\infty}$ \\
\hline $45.5 \mathrm{~km}(\mathrm{C} 1)$ & 1.30 & 274 & $2.10 \times 10^{-6}$ & 8.79 & 4.0 & 10.86 \\
$54.0 \mathrm{~km}(\mathrm{C} 2)$ & 0.43 & 270 & $7.28 \times 10^{-7}$ & 18.8 & 6.0 & 16.41 \\
$63.0 \mathrm{~km}(\mathrm{C} 3)$ & 0.15 & 270 & $2.60 \times 10^{-7}$ & 32.8 & 8.0 & 21.89 \\
CEV & 0.10 & 300 & $1.497 \times 10^{-7}$ & 50.8 & 10.0 & 25.96
\end{tabular}

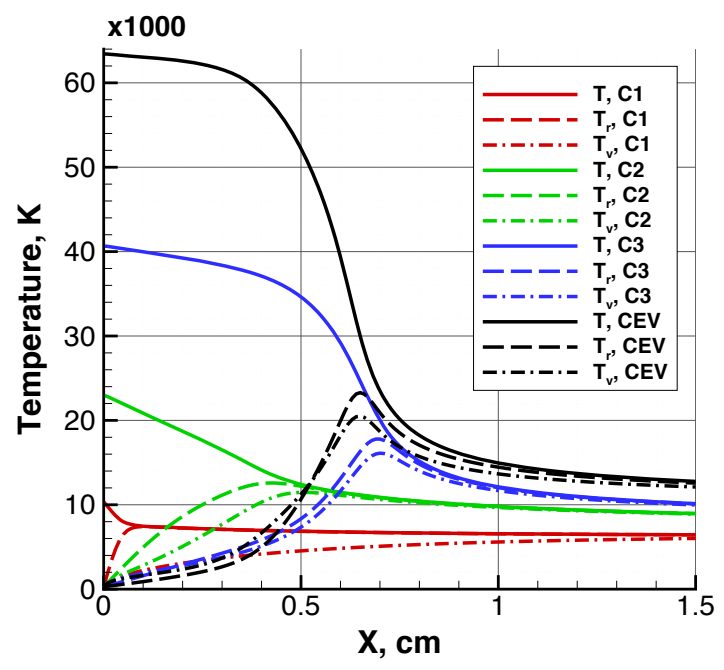

(a) Rotational and vibrational temperatures.

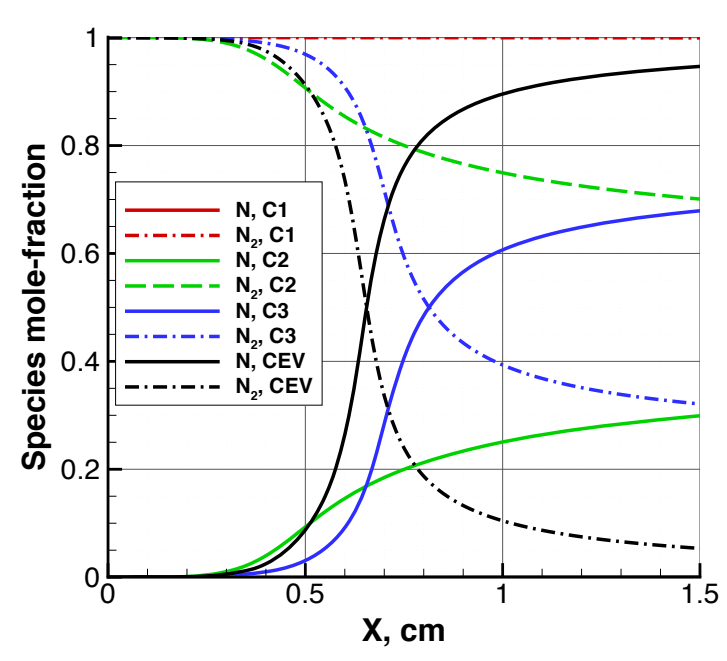

(b) Species mole-fractions.

Figure 10. Rotational and vibrational temperatures and species mole-fractions for 1-D post-normal shock calculations.

rotational relaxation model ${ }^{11}$ above $10,000 \mathrm{~K}$. In the present work for $\mathrm{N}+\mathrm{N}_{2}$ and the previous $\mathrm{H}_{2}$ studies, the vibrational and rotational relaxation times become identical when the temperature increases. In the present work, the collision limiting cross section is modified as a constant of $3.0 \times 10^{18} \mathrm{~cm}^{2}$ to satisfy these relaxation patterns. This cross section is determined through comparisons with the shock-tube data of the Millikan and White ${ }^{21}$ and Appleton. ${ }^{31}$

In the present work, a one-dimensional post-normal shock flow code is developed by using Eqs. (27) to (40). This flow code is constructed in two-parts; in the first part, the downstream conditions are determined by using the Rankine-Hugoniot relation for a frozen flow. In the second part, thermochemical nonequilibrium flows are calculated by the coupled equations of the one-dimensional flow and the system of master equations.

In Fig. 9, comparisons of the present rotational and vibrational temperatures and species mole-fractions with the shock-tube experiments by Sharma and Gillespie ${ }^{9}$ and $\mathrm{AVCO}^{32}$ and with the calculated results of the standard 2-temperature model are presented. In the 1-D post-normal shock calculations, the ambient pressure is set to 1.0 Torr and the shock velocity is $6.2 \mathrm{~km} / \mathrm{sec}$. The free-stream temperature is $300 \mathrm{~K}$. In this case, the total enthalpy of the free-stream is about $20 \mathrm{MJ} / \mathrm{kg}$. In figure (a), in the present calculation, the translational temperature behind the normal shock is about $24,300 \mathrm{~K}$ and the rotational and vibrational temperatures agree well with the measured temperatures by Sharma and Gillespie. After converging to the equilibrium temperature, where the distance is $0.3 \mathrm{~cm}$, the present temperature is slightly underestimated compared to the AVCO data. This underestimation is produced from many uncertainties, like the assumption of electronic ground state of $\mathrm{N}_{2}$ and from the omission of electrons and ionization. However, the present results show more reasonable results for the rotational and vibrational nonequilibrium in the post-normal shock flows than the results of the two-temperature model. In the two-temperature model, the transrotational temperature behind the post-normal shock is about $21,000 \mathrm{~K}$ and the vibrational temperature is signifcantly higher than measured vibrational temperature. In figure (b), it is shown that the dissociation of 
$\mathrm{N}_{2}$ rapidly occurs from $0.1 \mathrm{~cm}$ to $0.3 \mathrm{~cm}$ in the present calculation. This is because collision of $\mathrm{N}$ triggers the dissociation of $\mathrm{N}_{2}$ much more than the collision of $\mathrm{N}_{2}$. However, in the results from the two-temperature model, the dissociation of $\mathrm{N}_{2}$ occurs continuously.

In Table 3, initial conditions of several post-normal shock flows are tabulated. The free-stream conditions of cases $\mathrm{C} 1$ to $\mathrm{C} 3$ are constructed to be similar to the high-speed return conditions at altitudes from $45.5 \mathrm{~km}$ to $63.0 \mathrm{~km}$ of the Earth. In the present work, the simplification of pure $\mathrm{N}_{2}$ without electronic excitation, ionization and radiative heating is adopted to calculate the post-normal shock flows. While this is not an accurate re-entry calculation, it is enough to observe the rotational nonequilibrium of $\mathrm{N}_{2}$ in each re-entry condition. In the present work, the post-normal shock flow for a CEV condition is also calculated.

In Fig. 10, the temperatures and mole-fractions of the post-normal shock calculations are presented. In the $\mathrm{C} 1$ case, the rotational temperature rapidly approaches the translational temperature. Hence, in the $\mathrm{C} 1$ case, the rotational mode can be treated to be in equilibrium with the translational energy. However, for the $\mathrm{C} 2$, C3, and $\mathrm{CEV}$ cases, the rotational and vibrational nonequilibrium is obviously shown. In the $\mathrm{C} 2$ case, the rotational relaxation is slightly faster than the vibrational mode and in the C3 and CEV cases, the rotational and vibrational relaxations are almost identical. In figure (b), it is observed that the dissociation of $\mathrm{N}_{2}$ occurs rapidly, when the dissociated $\mathrm{N}$ is first generated. In figures (a) and (b), it is also observed that the heavy particle collisions by the dissociated $\mathrm{N}$ more significantly affects the rotational and vibrational relaxations of $\mathrm{N}_{2}$ than the collisions by $\mathrm{N}_{2}$ for the $\mathrm{C} 3$ and CEV cases.

\section{Conclusion}

Analyses using the master equation are performed for $\mathrm{N}+\mathrm{N}_{2}$ with the complete sets of the state-to-state transition rates from the NASA database. In the bound-bound transition studies, rotational nonequilibrium is clearly observed. Especially at temperatures above $30,000 \mathrm{~K}$, the relaxation rate of the rotational mode is almost identical with that of the vibrational mode. In the bound-free transition studies, it is shown that the dissociation of $\mathrm{N}_{2}$ occurs in the quasi-steady state of rotational and vibrational energies. However, recombination occurs in an equilibrium of the rotational and vibrational modes. The dissociation and recombination rate coefficients are determined from the results of the master equation calculations and the QSS assumption. These calculated reaction rate coefficients agree well with shock-tube experimental data. For the energy loss due to dissociation, it is observed that the normalized rotational and vibrational energy losses approach constants of 0.4 and 0.5 , when the temperature increases. The system of master equations are coupled with one-dimensional flow equations to analyze the relaxation of $\mathrm{N}_{2}$ in post-normal shock flows. In the comparisons with the shock-tube experiments, the present model approximately reproduces the measured rotational and vibrational temperatures. In the post-normal shock calculations for various re-entry conditions, the results lead to important conclusion that the rotational mode of $\mathrm{N}_{2}$ needs to be treated as a separate nonequilibrium mode in high speed re-entry calculations.

\section{Acknowledgments}

The authors gratefully acknowledge funding for this work through Air Force Office of Scientific Research Grant FA-9550-11-1-0309.

\section{References}

\footnotetext{
${ }^{1}$ Park, C., Nonequilibrium Hypersonic Aerothermodynamics, Wiley, New York, 1990.

${ }^{2}$ Kim, J. G., Kwon, O. J., and Park, C., "Master Equation Study and Nonequilibrium Chemical Reactions for $\mathrm{H}+\mathrm{H}_{2}$ and $\mathrm{He}+\mathrm{H}_{2}, "$ Journal of Thermophysics and Heat Transfer, Vol. 23, No. 3, 2009, pp. 443-453.

${ }^{3}$ Kim, J. G., Kwon, O. J., and Park, C., "Master Equation Study and Nonequilibrium Chemical Reactions for Hydrogen Molecule," Journal of Thermophysics and Heat Transfer, Vol. 24, No. 2, 2010, pp. 281-290.

${ }^{4}$ Kim, J. G. and Boyd, I. D., "Master Equation Study of Hydrogen Relaxation Using Complete Sets of State-to-state Transition Rates," AIAA Paper 2012-362, 50th AIAA Aerospace Sciences Meeting and Exhibit, Nashville, Tennessee, 2012.

${ }^{5}$ Parker, J. G., "Rotational and Vibrational Relaxation in Diatomic Gases," Physics of Fluids, Vol. 2, No. 4, 1959, pp. 449-462.

${ }^{6}$ Lordi, J. A. and Mates, R. E., "Rotational Relaxation in Nonpolar Diatomic Gas," Physics of Fluids, Vol. 13, No. 2, 1970, pp. 291-308.

${ }^{7}$ Carnevale, E. H., Carey, C., and Larson, G., "Ultrasonic Determination of Rotational Collision Numbers and Vibrational Relaxation Times of Polyatomic Gases at High Temperatures," Journal of Chemical Physics, Vol. 47, No. 8, 1967, pp. $2829-2835$.
} 
${ }^{8}$ Prangsma, G. J., Alberga, A. H., and Beenakker, J. J. M., "Ultrasonic Determination of the Volume Viscosity of $\mathrm{N}_{2}$, $\mathrm{CO}, \mathrm{CH}_{4}$, and $\mathrm{CD}_{4}$ Between 77 and 300K," Physica, Vol. 64, No. 2, 1973, pp. 278-288.

${ }^{9}$ Sharma, S. P. and Gillespie, W., "Nonequilibrium and Equilibrium Shock Front Radiation Measurement," Journal of Thermophysics and Heat Transfer, Vol. 5, No. 3, 1991, pp. 257-265.

${ }^{10}$ Fujita, K., Sato, S., Abe, T., and Ebinuma, Y., "Experimental Investigation of Air Radiation Behind a Strong Shock Wave," Journal of Thermophysics and Heat Transfer, Vol. 16, No. 1, 2002, pp. 77-82.

${ }^{11}$ Park, C., "Rotational Relaxation of $\mathrm{N}_{2}$ Behind a Strong Shock Wave," Journal of Thermophysics and Heat Transfer, Vol. 18, No. 4, 2004, pp. 527-533.

${ }^{12}$ Huo, W. M. and Green, S., "Quantum Calculations for Rotational Energy Transfer in Nitrogen Molecule Collisions," Journal of Chemical Physics, Vol. 109, No. 19, 1996, pp. 7572-7589.

${ }^{13} \mathrm{Sitz}$, G. O. and Farrow, R. L., "Pump-Probe Measurements of State-to-State Rotational Energy Transfer Rates in $\mathrm{N}_{2}(\mathrm{v}=1)$," Journal of Chemical Physics, Vol. 93, No. 11, 1990, pp. 7883-7893.

${ }^{14}$ Sharafutdinov, R. G., Belikov, A. E., Strekalov, M. L., and Storozhev, A. V., "State-to-State Rate Constants and Rotational Relaxation Time in Nitrogen," Chemical Physics, Vol. 207, No. 1, 1996, pp. 193-201.

${ }^{15}$ Jaffe, R., Schwenke, D., Chaban, G., and Huo, W. M., "Vibrational and Rotational Excitation and Relaxation of Nitrogen from Accurate Theoretical Calculation," AIAA paper 2008-1208, 46th AIAA Aerospace Sciences Meeting and Exhibit, Reno, Nevada, 2008.

${ }^{16}$ Jaffe, R., Schwenke, D., and Chaban, G., "Theoretical Analysis of $\mathrm{N}_{2}$ Collisional Dissociation and Rotation-Vibration Energy Transfer," AIAA paper 2009-1569, 47th AIAA Aerospace Sciences Meeting and Exhibit, Orlando, Florida, 2009.

${ }^{17}$ Gill, S., "A Process for The Step-by-step Integration of Differential Equations in An Automatic Digital Computing Machine," Mathematical Proceedings of the Cambridge Philosophical Society, Vol. 47, No. 1, 1951, pp. 96-108.

${ }^{18}$ Bulirsch, R. and Stoer, J., "Numerical Treatment of Ordinary Differential Equation by Extrapolation Method," $N u$ merische Mathematik, Vol. 8, No. 1, 1965, pp. 1-13.

${ }^{19}$ Park, C., "Review of Chemical-Kinetic Problems of Future NASA Missions, I: Earth Entries," Journal of Thermophysics and Heat Transfer, Vol. 7, No. 3, 1993, pp. 385-398.

${ }^{20}$ Landau, L. and Teller, E., "Theory of Sound Dispersion," Physics Sowjetunion, Vol. 10, 1936, pp. 34-43.

${ }^{21}$ Millikan, R. C. and White, D. R., "Systematics of Vibrational Relaxation," Journal of Chemical Physics, Vol. 39, No. 12, 1963, pp. 3209-3213.

${ }^{22}$ Cary, B., "Shock-Tube Study of the Thermal Dissociation of Nitrogen," Physics of Fluids, Vol. 8, No. 1. 1965, pp. 26-35.

${ }^{23}$ Byron, S., "Shock-Tube Measurement of the Rate of Dissociation of Nitrogen," Journal of Chemical Physics, Vol. 44, No. 4, 1966, pp. 1378-1388.

${ }^{24}$ Appleton, J. P., Steinberg, M., and Liquornik, D. J., "Shock-Tube Study of Nitrogen Dissociation using VacuumUltraviolet Light Absorption," Journal of Chemical Physics, Vol. 48, No. 2, 1968, pp. 599-608.

${ }^{25}$ Hanson, R. K. and Baganoff, D., "Shock-Tube Study of Nitrogen Dissociation Rates Using Pressure Measurements," AIAA Journal, Vol. 10, No. 2, 1972, pp. 211-215.

${ }^{26}$ Allen, R. A., Keck, J. C., and Camm, J. C., "Nonequilibrium Radiation and the Recombination Rate of Shock-Heated Nitrogen," Physics of Fluids, Vol. 5, No. 3, 1962, pp. 284-291.

${ }^{27}$ Hammerling, P., Teare, J. D., and Kivel, B., "Theory of Radiation from Luminous Shock Waves in Nitrogen," Physics of Fluids, Vol. 2, No. 4, 1959, pp. 422-426.

${ }^{28}$ Lin, S. C. and Fyfe, W. I., "Low-Density Shock Tube for Chemical Kinetics Studies," Physics of Fluids, Vol. 4, No. 2, 1961, pp. 238-249.

${ }^{29}$ Baulch, D. L., Drysdale, D. D., and Horne, D. G, Evalulated Kinetic Data For High Temperature Reactions, Volume 2, CRC Press, London, 1973.

${ }^{30}$ Rahn, L. A. and Palmer, R. E., "Studies of Nitrogen Self-Broadening at High Temperature with Inverse Raman Spectroscopy," Journal of Optical Society of America, Vol. 3, No. 9, 1986, pp. 1164-1169.

${ }^{31}$ Appleton, J. P., "Shock-Tube Study of the Vibrational Relaxation of Nitrogen Using Vacuum-Ultraviolet Light Absorption," Journal of Chemical Physics, Vol. 47, No. 9, 1967, pp. 3231-3240.

32 Allen, R. A., "Nonequilibrium Shock Front Rotational, Vibrational, and Electronic Temperature Measurements," AVCO Everett Research Lab., Everett, MA, Research Rept. 186, Aug. 1964. 